\title{
A Modified Monte-Carlo Quadrature
}

\section{By Seymour Haber}

1. Introduction. The simple Monte-Carlo method for approximately evaluating an integral $I=\int_{A} f d v$, where $A$ is a region in $k$-dimensional Euclidean space and $d v$ is the volume element, is as follows: For some integer $N$, points $x_{1}, x_{2}, \cdots, x_{N}$ are chosen at random (i.e., with uniform distribution) in $A$ and the integral is estimated by the quantity

$$
J=|A| \cdot \frac{f\left(x_{1}\right)+\cdots+f\left(x_{N}\right)}{N},
$$

where " $|A|$ " denotes the ( $k$-dimensional) volume of $A$. If the $x_{i}$ are regarded as independent (or at least pairwise independent) random variables, then the estimator $J$ is a random variable whose mean is $I$ and whose standard deviation is $d N^{-1 / 2}$, where $d^{2}=|A| \int_{A} f^{2}-\left(\int_{A} f\right)^{2}$. ( $I$ is the mean, and $d$ is the standard deviation of the random variable $|A| f(x)$, where $x$ is a random variable uniformly distributed on $A$.) The standard deviation of $J$ is taken as a measure of the error to be expected in taking a sample value of $J$, as above, as an estimate of $I$. The error thus decreases very slowly as $N$ (and the expense of the calculation) increases and may be unacceptably large even for quite high values of $N$. As a result, a great deal of effort has gone into devising more sophisticated forms of Monte-Carlo procedures (see, e.g., [1]) in order to replace $J$ by estimators of lower variance. Each of these methods involves adaptation of the computation procedure to the particular function being integrated; thus it necessitates preliminary analysis of the integrand and the writing of a special integration program. (Monte-Carlo calculations are generally done on automatic computers.) In this paper we present a modified Monte-Carlo quadrature method whose application is completely automatic and which produces an estimate of $I$ whose variance is slightly, but often significantly, lower than that of $J$.

2. The Procedure. Our method is a form of stratified sampling [2]; however the region $A$ (the "population") is broken into subregions defined simply in terms of its geometry, rather than in terms of the values of $f$ we expect to find in them. We define the procedure in the case that $A$ is a $k$-dimensional interval, i.e. the cartesian product of one-dimensional intervals $\left(a^{i}, b^{i}\right), i=1, \cdots, k$. (By using other than cartesian coordinates, the method may be applied to regions of some other shapes.) Each interval $\left(a^{i}, b^{i}\right)$ is divided into subintervals by the subdivision points $a^{i}=a_{i, 0}<$ $a_{i, 1}<\cdots<a_{i, n_{i}}=b^{i}$. This is done in such a way that for each $i$ the differences $a_{i, j+1}-a_{i, j}$ are commensurable; most simply, all of them may be multiples of the smallest one. The interval $A$ is thus subdivided into $n=n_{1} \cdot n_{2} \cdot \cdots \cdot n_{k}$ subintervals defined by inequalities of the form $a_{i, j}<x^{i}<a_{i, j+1}$. (It is of no significance whether we use strict inequalities, as written, or loose; the points left out form a set of ( $k$-dimensional Lebesgue) measure zero.) Calling these subintervals, in some order, $A_{1}, A_{2}, \cdots, A_{n}$, we observe that since their volumes are commensurable there is a least number $\alpha$ such that $\alpha \cdot\left|A_{i}\right|$ is an integer $p_{i}$ for all $i$, and so if $N=$

Received August 11, 1965. 
$\left(p_{1}+\cdots+p_{n}\right) m, m$ an integer, it is possible to distribute $N$ points $x_{1}, \cdots, x_{N}$ in $A$ in such a way that the number of points found in each $A_{i}$ is proportional to $\left|A_{i}\right|$. We do this by fixing $m$, and therefore $N$, and then choosing $m \cdot p_{i}$ points at random in $A_{i}$, for each $i$; and we take as our estimate of $I$ the quantity

$$
J^{\prime}=\frac{|A|}{N} \sum_{i=1}^{N} f\left(x_{i}\right) .
$$

The simplest, and in most cases probably the best, form of this procedure, is to divide each interval $\left(a_{i}, b_{i}\right)$ into equal subintervals (though the subintervals for one $i$ need not be equal in length to those for another $i$ ). Then the $A_{i}$ are all congruent and we can just choose one point in each $A_{i}$ and then calculate $J^{\prime}$. In this case $m=1$ and $p_{i}=1$ for each $i$ and $N=n$. When referring to this case, we shall denote the estimate of $I$ by $J^{\prime \prime}$; so that anything said below about $J^{\prime}$ applies also to $J^{\prime \prime}$, but not conversely.

Theorem 1, below, is a form of a standard result in the theory of sampling; see, e.g., [2]. A proof is given here for the sake of completeness.

3. Error Analysis. In each $A_{i}, i=1, \cdots, n$, we choose at random $m \cdot p_{i}$ points $x_{i, 1}, x_{i, 2}, \cdots, x_{i, m} \cdot p_{i}$. Treating the points as independent random variables, with each $x_{i, j}$ uniformly distributed over $A_{i}$, we have (" $m(\cdot)$ ") and " $\sigma^{2}(\cdot)$ ") denoting, as usual, the mean and variance of random variables) :

$$
\begin{aligned}
& m\left(f\left(x_{i, j}\right)\right)=\frac{1}{\left|A_{i}\right|} \int_{A_{i}} f, \quad j=1, \cdots, m \cdot p_{i}, \\
& \sigma^{2}\left(f\left(x_{i, j}\right)\right)=\frac{1}{\left|A_{i}\right|}\left(\int_{A_{i}} f^{2}-\frac{1}{\left|A_{i}\right|}\left(\int_{A_{i}} f\right)^{2}\right) .
\end{aligned}
$$

We shall call these last quantities " $m_{i}$ " and " $\sigma_{i}{ }^{2}$ ". Now

and so

$$
J^{\prime}=\frac{|A|}{N} \sum_{i=1}^{n} \sum_{j=1}^{m \cdot p_{i}} f\left(x_{i, j}\right)
$$

$$
m\left(J^{\prime}\right)=\frac{|A|}{N} \sum_{i=1}^{n} \frac{m \cdot p_{i}}{\left|A_{i}\right|} \int_{A_{i}} f=\int_{A} f
$$

since $p_{i} /\left|A_{i}\right|=\alpha=N /(m \cdot|A|)$. Also,

$$
\sigma^{2}\left(J^{\prime}\right)=\frac{|A|^{2}}{N^{2}} \sum_{i=1}^{n} m \cdot p_{i} \cdot \sigma_{i}^{2} .
$$

The variance of the simple Monte-Carlo estimator $J$, with an equal number of points $N$, is

$$
\sigma^{2}(J)=\frac{1}{N}\left(|A| \int_{A} f^{2}-\left(\int_{A} f\right)^{2}\right) .
$$

Now $m \cdot p_{i}=N \cdot\left|A_{i}\right| /|A|$ and so

$$
\begin{aligned}
\sigma^{2}\left(J^{\prime}\right) & =\frac{|A|}{N} \sum_{i=1}^{n}\left|A_{i}\right| \sigma_{i}{ }^{2}=\frac{|A|}{N} \sum_{i=1}^{n}\left(\int_{A_{i}} f^{2}-\frac{1}{\left|A_{i}\right|}\left(\int_{A_{i}} f\right)^{2}\right) \\
& =\frac{|A|}{N}\left(\int_{A} f^{2}-\sum_{i=1}^{n}\left|A_{i}\right| m_{i}{ }^{2}\right) .
\end{aligned}
$$


Thus

$$
\begin{aligned}
N\left(\sigma^{2}(J)-\sigma^{2}\left(J^{\prime}\right)\right) & =|A| \sum_{i=1}^{n}\left|A_{i}\right| m_{i}{ }^{2}-I^{2} \\
& =|A| \sum_{i=1}^{n}\left|A_{i}\right|\left(m_{i}-\frac{I}{|A|}\right)^{2}
\end{aligned}
$$

which is nomnegative, and we have proved:

Theorem 1. For any integrand (in $\left.L^{2}(A)\right)$

$$
\sigma^{2}\left(J^{\prime}\right) \leqq \sigma^{2}(J),
$$

when the integrand is evaluated at the same number of points in the calculation of $J$ and of $J^{\prime}$.

Thus there is never any loss of expected accuracy in replacing simple MonteCarlo quadrature by the method proposed. In fact, the two standard deviations are equal only if $\left(1 /\left|A_{i}\right|\right) \int_{A_{i}} f$ is independent of $i$, which would certainly occur only rarely, so that generally we can expect a gain in accuracy.

For the simple version (where we denote the estimate by $J^{\prime \prime}$ ) we can say more, if the integrand is continuous and if we restrict the shapes of the subintervals in a certain way.

Definition [3]. If $A$ is a $k$-dimensional interval $\prod_{i=1}^{k}\left(a^{i}, b^{i}\right)$ the "modulus of regularity" of $A$ is the quantity

$$
\rho(A)=|A| / \max _{1 \leqq i \leqq k}\left(b^{i}-a^{i}\right)^{k} .
$$

A collection of intervals is called "regular" if there is a number $\rho_{0}>0$ such that $\rho(A) \geqq \rho_{0}$ for every interval $A$ in the collection.

THEOREM '2. If the integrand is continuous on the closed interval $A$, and the collection of subintervals used in calculating $J^{\prime \prime}$ for a sequence of values of $N$ going to infinity is regular, then $\sigma\left(J^{\prime \prime}\right) / \sigma(J) \rightarrow 0$ as $N \rightarrow \infty$ through the sequence.

Proof. Since for each $N$ the subintervals are all congruent and so of volume | $A \mid / N$, the regularity condition implies that as $N$ goes to infinity the diameters of the subintervals approach zero uniformly. $f$ is uniformly continuous, so the quantity

$$
\sigma_{i}^{2}=\frac{1}{\left|A_{i}\right|} \int_{A_{i}}\left(f-\frac{1}{\left|A_{i}\right|} \int_{A_{i}} f\right)^{2}
$$

goes to zero uniformly in $i$. Thus for any positive $\epsilon$ there is an $N(\epsilon)$ so large that for $N \geqq N(\epsilon)$

$$
\sigma^{2}\left(J^{\prime \prime}\right)=\frac{|A|}{N} \sum_{i=1}^{N}\left|A_{i}\right| \sigma_{i}{ }^{2}=\frac{|A|^{2}}{N} \cdot \frac{\sigma_{1}{ }^{2}+\cdots+\sigma_{N}{ }^{2}}{N}<\epsilon \frac{|A|^{2}}{N}
$$

so that $\sigma\left(J^{\prime \prime}\right)=o\left(N^{-1 / 2}\right)$, while $\sigma(J) / N^{-1 / 2}=d>0$.

So we see that under the hypotheses of Theorem 2 the expected error actually goes to zero faster than $N^{-1 / 2}$. The continuity hypothesis can be greatly relaxed. If the integrand is bounded, and its discontinuities all lie on a few smooth $(k-1)$-dimensional surfaces in $A$ (as is usual when the integrand comes from a physical problem), the conclusion still holds. For then if $\epsilon$ is any positive number, there is an open subset $B$ of $A$, of measure at least $A-\epsilon$, on which $f$ is uniformly continuous. For $N$ sufficiently large at least $N(1-2 \epsilon)$ of the $A_{i}$ lie entirely in $B$ and the above 
reasoning may be applied to them; and the remaining $A_{i}$ cannot contribute more than $C \epsilon N^{-1 / 2}$ to $\sigma\left(J^{\prime \prime}\right)$, for some constant $C$ depending on the bound of $f$.

However the increase in the speed of convergence is not great. By imposing further smoothness conditions on the integrand we can obtain a precise result:

Theorem 3. Under the hypotheses of Theorem 2 , if $f$ is also differentiable and its gradient is continuous and bounded on $A$, then there are positive numbers $C_{1}$ ! and $C_{2}$, independent of $f$, such that for any $\epsilon>0$

$$
\begin{aligned}
\left(C_{2}-\epsilon\right) \frac{|A|^{1+1 / k}}{12^{1 / 2}}\left(\frac{1}{|A|} \int_{A}|\nabla f|^{2}\right)^{1 / 2} N^{-1 / 2-1 / k} & \leqq \sigma\left(J^{\prime \prime}\right) \\
& \leqq\left(C_{1}+\epsilon\right) \frac{|A|^{1+1 / k}}{12^{1 / 2}}\left(\frac{1}{|A|} \int_{A}|\nabla f|^{2}\right)^{1 / 2} N^{-1 / 2-1 / k}
\end{aligned}
$$

for all sufficiently large $N$. If all the subintervals used in the calculation of $J^{\prime \prime}$ are ( $k$-dimensional) cubes, then $C_{1}=C_{2}=1$.

Proof. Let $w_{i}=\left(w_{i}{ }^{1}, \cdots, w_{i}{ }^{k}\right)$ be the center of the subinterval $A_{i}$. Let $c_{i}=f\left(w_{i}\right), d_{i}^{j}=\left(\partial f / \partial x^{j}\right)\left(w_{i}\right)$. Then throughout $A_{i}$

$$
f(x)=c_{i}+\sum_{j=1}^{k} d_{i}^{j}\left(x^{j}-w^{j}\right)+o\left(\sum_{j=1}^{k}\left(x^{j}-w^{j}\right)^{2}\right)^{1 / 2} .
$$

(Here and below the symbol " $o(\cdot)$ " refers to what happens as $N \rightarrow \infty$ and the diameters of the $A_{i}$ shrink to zero.) We can write

$$
\int_{A_{i}} f^{2}-\frac{1}{\left|A_{i}\right|}\left(\int_{A_{i}} f\right)^{2}
$$

as

$$
\int_{A^{\prime}}\left[c_{i}+\sum_{j=1}^{k} d_{i}{ }^{j} x^{j}+o(r)\right]^{2} d x-\frac{1}{\left|A^{\prime}\right|}\left(\int_{A^{\prime}}\left[c_{i}+\sum_{j=1}^{k} d_{i}{ }^{j} x^{j}+o(r)\right] d x\right)^{2},
$$

where $A^{\prime}=\prod_{j=1}^{k}\left(a^{j}, b^{j}\right)$ is the region obtained from $A_{i}$ by the translation taking $w_{i}$ into the origin, and $r$ is the distance of $x$ from the origin.

Now

$$
\int_{\Lambda^{\prime}} r^{2} d x=\sum_{j=1}^{k} \int_{A^{\prime}}\left(x^{i}\right)^{2} d x=\frac{\left|A^{\prime}\right|}{12} \sum_{j=1}^{k}\left(b^{i}-a^{i}\right)^{2}
$$

Let $\rho$ be a lower bound for the moduli of regularity of all the subintervals $A_{i}$ used for the various values of $N$ in the sequence under consideration. Then

$$
\max _{1 \leqq j \leqq k}\left(b^{i}-a^{i}\right)^{2} \leqq\left(\rho^{-1}\left|A^{\prime}\right|\right)^{2 / k}
$$

Since for any fixed $N,\left|A_{i}\right|=\left|A^{\prime}\right|=|A| / N$, we have

$$
\int_{A^{\prime}} r^{2} d x \leqq \frac{k \rho^{-2 / k}|A|^{1+2 / k}}{12 N^{1+2 / k}}
$$

It follows that

$$
\int_{A^{\prime}} o(r) \leqq\left(\int_{A^{\prime}}(o(r))^{2}\right)^{1 / 2}\left(\int_{A^{\prime}} 1\right)^{1 / 2}=o\left(\frac{|A|}{N} \int_{A^{\prime}} r^{2}\right)^{1 / 2}=o\left(N^{-1-1 / k}\right) ;
$$


and also that

$$
\int_{A^{\prime}}\left(\sum_{j=1}^{k} d_{i}^{j} x^{j}\right)(o(r)) \leqq\left[\int_{A^{\prime}}\left(\sum_{j=1}^{k} d_{i}^{j} x^{j}\right)^{2} \int_{A^{\prime}}(o(r))^{2}\right]^{1 / 2}=o\left(N^{-1-2 / k}\right),
$$

since

$$
\begin{aligned}
\int_{A^{\prime}}\left(\sum_{j=1}^{k} d_{i}{ }^{j} x^{j}\right)^{2} & =\int_{A^{\prime}} \sum_{j=1}^{k}\left(d_{i}^{j} x^{j}\right)^{2}=\frac{\left|A^{\prime}\right|}{12} \sum_{j=1}^{k}\left(d_{i}^{j}\right)^{2}\left(b^{j}-a^{j}\right)^{2} \\
& \leqq\left|\nabla f\left(w_{i}\right)\right|^{2} \frac{\rho^{-2 / k}\left|A^{\prime}\right|^{1+2 / k}}{12}
\end{aligned}
$$

Expanding out (2) and making use of these estimates, we obtain:

$$
\int_{A_{i}} f^{2}-\frac{1}{\left|A_{i}\right|}\left(\int_{A_{i}} f\right)^{2}=\frac{|A|}{12 N} \sum_{j=1}^{k}\left(d_{i}^{j}\right)^{2}\left(b^{j}-a^{j}\right)^{2}+o\left(N^{-1-2 / k}\right) .
$$

Now

$$
\begin{gathered}
\left|\nabla f\left(w_{i}\right)\right|^{2} \min \left(b^{j}-a^{j}\right)^{2} \leqq \sum_{j=1}^{k}\left(d_{i}^{j}\right)^{2}\left(b^{j}-a^{j}\right)^{2} \leqq\left|\nabla f\left(w_{i}\right)\right|^{2} \max \left(b^{j}-a^{j}\right)^{2}, \\
\frac{\left|A^{\prime}\right|}{\max \left(b^{j}-a^{j}\right)^{k-1}} \geqq \rho \max \left(b^{j}-a^{j}\right),
\end{gathered}
$$

while

$$
\begin{aligned}
\min \left(b^{j}-a^{j}\right) & \geqq \frac{\left|A^{\prime}\right|}{\max \left(b^{j}-a^{j}\right)^{k-1}} \geqq\left|A^{\prime}\right|^{1 / k}\left(\frac{\left|A^{\prime}\right|^{1 / k}}{\max \left(b^{j}-a^{j}\right)}\right)^{k-1} \\
& \geqq \rho^{1-1 / k}\left|A^{\prime}\right|^{1 / k} ;
\end{aligned}
$$

so that

$$
\rho^{2}\left(\frac{\rho^{-1}|A|}{N}\right)^{2 / k}\left|\nabla f\left(w_{i}\right)\right|^{2} \leqq \sum_{j=1}^{k}\left(d_{i}^{j}\right)^{2}\left(b^{j}-a^{j}\right)^{2} \leqq\left(\frac{\rho^{-1}|A|}{N}\right)^{2 / k}\left|\nabla f\left(w_{i}\right)\right|^{2} .
$$

We now set $C_{1}=\rho^{-2 / k}, C_{2}=\rho^{2-2 / k}$, and note that

$$
\sum_{i=1}^{N}\left|A_{i}\right|\left|\nabla f\left(w_{i}\right)\right|^{2} \rightarrow \int_{A}|\nabla f|^{2} \text { as } N \rightarrow \infty .
$$

The theorem now follows from (1), (3), (4), and (5).

The proof indicates that, in a case where it is known that the integrand varies more rapidly with certain of the variables than with others (i.e. certain partial derivatives $\partial f / \partial x^{i}$ are generally greater in magnitude throughout $A$ than others) it would be best to subdivide the edges of $A$ so that $b^{j}-a^{j}$ is approximately inversely proportional to $\partial f / \partial x^{j}$, on the average. This would incorporate a weak form of importance sampling into the calculation of $J^{\prime \prime}$, and would naturally require a preliminary analysis of the integrand. In the absence of such analysis it seems that it would be best generally to so subdivide $A$ as to make the $b^{j}-a^{j}$ nearly equal. This would put $\rho$ near one, and so minimize the constant $C_{1}$ in the upper bound for $\sigma\left(J^{\prime \prime}\right)$.

If $\rho$ is close to one, then $\sigma\left(J^{\prime \prime}\right) / \sigma\left(J^{\prime}\right)$ will, for large values of $N$, be approximately 
equal to $\left(d^{\prime \prime} / d\right) N^{-1 / k}$, where

$$
d^{\prime \prime}=\frac{|A|^{1+1 / k}}{12^{1 / 2}}\left(\frac{1}{|A|} \int_{A}|\nabla f|^{2}\right)^{1 / 2} .
$$

$d^{\prime \prime}$ is a measure of the variability of $f$ on $A$, just as is the variance $d$. If $A$ is a cube and $f$ is linear, $d^{\prime \prime}=d$. Otherwise $d^{\prime \prime} / d$ may be larger or smaller than one.

4. Experiments. Tables I and II display the results of the calculation of two integrals by the simple and modified Monte-Carlo methods on the IBM 7094 computer of the National Bureau of Standards. In both cases the dimension $k$ was 4 , and the region of integration $A$ was the unit cube. In the modified Monte-Carlo calculations $A$ was partitioned into cubes by partitioning each of its edges into the same number of equal subintervals. In each table $N$ is the number of points used, $E_{1}$ is the error of the simple Monte-Carlo calculation and $E_{2}$ that of $J^{\prime \prime} . r_{1}$ is the ratio of $\left|E_{1}\right|$ to the probable error (at the $50 \%$ level), which is taken as $.6745 \sigma(J) ; r_{2}$ is, similarly, the ratio of $\left|E_{2}\right|$ to that quantity. In Table II $r_{3}$ is the ratio of $\left|E_{2}\right|$ to the probable error of $J^{\prime \prime}$ that would be indicated by Theorem 3, i.e. to the quantity $.6745 l^{\prime \prime} N^{-3 / 4}$. The pseudo-random points used for each calculation were the points

$x_{n}=\left(\left\{\frac{n(n+1)}{2} 2^{1 / 2}\right\},\left\{\frac{n(n+1)}{2} 3^{1 / 2}\right\},\left\{\frac{n(n+1)}{2} 5^{1 / 2}\right\},\left\{\frac{n(n+1)}{2} 7^{1 / 2}\right\}\right)$

where $\{a\}$ is the fractional part of $a$.

For table I the integrand was given by $f(x)=1$ if $\left(x^{1}\right)^{2}+\cdots+\left(x^{4}\right)^{2} \leqq 1$, $f(x)=0$ otherwise. The true value of the integral is .308425 , and $d=.462$. In Table II the integrand $f(x)=\exp \left[x^{1} x^{2} x^{3} x^{4}\right]-1$; the integral is $.0693976, d=.112$, $d^{\prime \prime}=.142$.

The first integrand is discontinuous, and the second is smooth. In each case the results of using the proposed method were better than those from simple MonteCarlo, but the improvement was greater in the second case. The last 2 columns of Table II show the results to be in fair agreement with what one would expect after Theorem 3.

I wish to thank Mr. Louis Joseph and Mr. Kenneth Kloss, of the National Bureau of Standards, for carrying out these calculations.

TABLE I

\begin{tabular}{|c|c|c|c|c|}
\hline$N$ & $E_{1}$ & $E_{2}^{\prime}$ & $r_{1}$ & $r_{2}$ \\
\hline 16 & .0584 & -.0666 & .74 & .86 \\
\hline 81 & -.0002 & -.0002 & .01 & .01 \\
\hline 256 & -.0119 & -.0080 & .61 & .41 \\
\hline 625 & -.0180 & .0012 & 1.45 & .10 \\
\hline 1,296 & -.0087 & -.0072 & 1.00 & .81 \\
\hline 2,401 & -.0085 & -.0023 & 1.35 & .37 \\
\hline 4,096 & -.0075 & -.0024 & 1.55 & .49 \\
\hline 6,561 & -.0064 & -.0008 & 1.67 & .21 \\
\hline 10,000 & -.0011 & .00122 & .35 & .39 \\
\hline 65,536 & .00052 & -.00037 & .42 & .31 \\
\hline
\end{tabular}


TABLE II

\begin{tabular}{r|c|c|c|c|c}
\multicolumn{1}{c|}{$N$} & $E_{1}$ & $E_{2}$ & $r_{1}$ & $r_{2}$ & \multicolumn{1}{c}{$r_{3}$} \\
\hline 16 & -.0067 & -.0033 & .36 & .17 & .27 \\
81 & -.0040 & .0003 & .48 & .04 & .09 \\
256 & -.0010 & -.0008 & .21 & .12 & .37 \\
625 & .0026 & .0024 & .86 & .81 & 3.18 \\
1,296 & -.0003 & -.00038 & .15 & .19 & .87 \\
2,401 & -.0015 & .00001 & .98 & .00 & .03 \\
4,096 & -.0009 & .00026 & .77 & .22 & 1.42 \\
6,561 & -.0014 & -.00008 & 1.55 & .01 & .05 \\
10,000 & .0012 & -.00022 & 1.64 & .29 & 2.34 \\
65,536 & -.00021 & .000026 & .73 & .09 & 1.04 \\
\hline
\end{tabular}

5. Discussion. A disadvantage of the proposed method is the restriction it imposes on the number of points $N$. $N$ must be a multiple of $n_{1} \cdot n_{2} \cdots \cdots n_{k}$; if $\rho$ is to be near one, the $n_{i}$ are themselves restricted in the values they may take. In the very simplest situation, where $A$ is a cube and the $A_{i}$ congruent subcubes as in the above calculations, $N$ must be one of the numbers $2^{k}, 3^{k}, 4^{k}, \cdots$; and even for moderate values of $k$ only a few of these values of $N$ are practicable. In the more general situation the restriction is not quite so tight. In all cases the number $N$ must be fixed in advance, and the calculation redone from the beginning if $N$ is changed. In simple Monte-Carlo, by contrast, if it is suspected that a calculation done with $N$ points did not result in sufficient accuracy, and a calculation with $N^{\prime}>N$ points is wanted, it is necessary only to calculate another $N^{\prime}-N$ points. In practice this last point is not apt to be very serious. Convergence is so slow in simple MonteCarlo that for a significant improvement in accuracy $N^{\prime}$ will have to be considerably larger than $N$, so that calculating $N^{\prime}$ points will not be much more expensive than calculating $N^{\prime}-N$.

Another disadvantage is that the expected error cannot be neatly estimated as in a simple Monte-Carlo calculation. In the latter, one can, by calculating $f^{2}$ together with $f$ at each point, get an estimate of $\int f^{2}$ and so of $d$ in the course of the calculation. There is no similarly inexpensive way of calculating $d^{\prime \prime}$. One might modify the calculation by choosing two points, $x_{i}$ and $x_{i}{ }^{\prime}$, in each subinterval $A_{i}$, and calculating the auxiliary quantity $\left(f\left(x_{i}\right)-f\left(x_{i}{ }^{\prime}\right)\right)^{2}$. Then

$$
\frac{|A|^{2}}{2 N^{2}} \sum_{i=1}^{N}\left[f\left(x_{i}\right)-f\left(x_{i}{ }^{\prime}\right)\right]^{2}
$$

is a good estimate of $\sigma^{2}\left(J^{\prime \prime}\right)$. However, taking the quantity

$$
\frac{|A|}{N} \sum_{i=1}^{N}\left[f\left(x_{i}\right)+f\left(x_{i}^{\prime}\right)\right] / 2
$$

as an estimate of the integral is equivalent to making two independent evaluations of $J^{\prime \prime}$ and averaging them. This reduces the variance by a factor of 2 ; so the estimated standard deviation of the last estimate of the integral would be

$$
\frac{|A|}{2 N}\left(\sum_{i=1}^{N}\left[f\left(x_{i}\right)-f\left(x_{i}{ }^{\prime}\right)\right]^{2}\right)^{1 / 2} \text {. }
$$


The extra computing required to place the random points in the $A_{i}$ comes to $2 k$ additions per point. This will be significant only if the integrand is very easy to calculate and $N$ is very large; and the larger $N$ is, the greater the advantage in accuracy of the present method.

It is of some interest to compare $J^{\prime \prime}$ with the nonrandom integration rule in which $f$ is evaluated at the center $w_{i}$ of each subinterval $A_{i}$, and $(|A| / N) \sum_{i=1}^{N} f\left(w_{i}\right)$ is taken as the approximate value of $I$. This is a $k$-dimensional "midpoint rule", and it can be shown that if the integrand has bounded second partial derivatives, the error will be asymptotic to $C N^{-2 / k}$, where $C$ is some constant depending on $f$. If the integrand satisfies only the hypotheses of Theorem 3, all that could be said, in general, is that the error would be $O\left(N^{-1 / k}\right)$. It thus appears that by choosing our point at random in the subinterval, rather than fixing it at the center, we gain the full strength of Monte-Carlo computation: the exponent $-\frac{1}{2}$, characteristic of Monte-Carlo, is added to the exponent $-1 / k$ which is characteristic of the midpoint rule (and in fact of any nonrandom rule) for the class of functions considered. This seems rather surprising, and an explanation is to be sought, we believe, in the consideration of the uniformity of distribution - in the sense discussed in [4]of the points at which the integrand is evaluated. It appears that the set of randomly chosen points is on the average better distributed, in a certain sense, than the set of the central points of the subintervals.

I wish to thank Dr. Joan R. Rosenblatt, of the National Bureau of Standards, for helpful comments and references to the statistical literature.

6. Conclusion. The last few years have seen a great decrease in the cost of very long computations. A further decrease, by one or two orders of magnitude, can be expected in the next five or ten years. When the Monte-Carlo procedure was first suggested, at the beginning of the automatic computer era, computation was relatively slow and expensive and the method could, in most cases, be successfully applied only if an analysis of the problem led to special adaptations which reduced the variance very greatly. Now, however, even simple Monte-Carlo is becoming increasingly practical. It seems desirable, at this time, to begin to design automatic procedures of the Monte-Carlo type which may be kept as "library routines" to be used, without much analysis, by any investigator needing the value of a multiple integral.

National Bureau of Standards

Washington, D. C.

1. H. KAHN, "Use of different Monte Carlo sampling techniques," Symposium on Monte Carlo Methods (University of Florida, 1954), Wiley, New York and Chapman and Hall, London, 1956 , pp. 146-190. MR 18, 151 .

2. W. G. Cochran, Sampling Techniques, Wiley, New York and Chapman and Hall, London, 1953; 2nd rev. enlarged ed., Wiley, New York, 1963, p. 98. MR 14, 887; MR 28 \#695.

3. S. SAKS, Théorie de l'Integral, Z Subwencji Funduszu Kultury Narodowej, Warsaw, 1933; English transl. of 2nd rev. ed., Monografie Matematyczne, Vol. VII, Stechert, New York, 1937; corrected reprint of English transl., Dover, New York, 1964, p. 106. MR $29 \# 4850$.

4. J. M. Hammersley, "Monte Carlo methods for solving multivariable problems," Ann. New York Acad. Sci., v. 86, 1960, pp. 844-874. MR $22 \# 8644$. 